\title{
Impact of high-speed trains in small isolated power system phase to phase imbalances
}

\author{
I. Saboya ${ }^{1}$, I. Egido $^{1}$, E. Pilo $^{2}$ \& L. Rouco ${ }^{1}$ \\ ${ }^{1}$ Instituto de Investigación Tecnológica, \\ Escuela Técnica Superior de Ingeniería (ICAI), \\ Universidad Pontificia Comillas, Madrid, Spain \\ ${ }^{2}$ EPRail Research and Consulting, Madrid, Spain
}

\begin{abstract}
High-speed trains are more and more widely used around the world. They are commonly installed in interconnected power systems by connecting each traction substation directly in different nodes among the network. The single-phase loads of high-speed railway systems do not produce excessive dangerous phase to phase imbalances in these robust networks. However, if high-speed trains are to be used in small isolated power systems, such as islands or non-interconnected power systems, problems of unacceptable phase to phase imbalances may appear. This is due to the small size of the system and its relatively weak network that lead to a low short-circuit power in their nodes. This paper studies the impact of high-speed trains in small isolated power systems. Two different connections proposed by the infrastructure manager are analyzed. These connections try to reduce the impact of the railway network in power system network imbalances. Both connection schemes have been tested in a real small isolated power system in which a high-speed railway system is expected to be installed by the year 2018. Representative peak and valley scenarios for this year have been used in the analysis. The results obtained evidence that small isolated power systems are very sensitive to the incorporation of high-speed trains in their weak networks since high imbalances are obtained. Moreover, the results allow the selection of the connection that is more capable of reducing power system imbalances.

Keywords: high-speed train, phase to phase imbalances, small isolated power system.
\end{abstract}




\section{Introduction}

Due to the amount of power which is involved, AC-fed high-speed railways are normally fed directly from the transmission network through transformers installed in the traction substations. In some countries, there is a traction transmission network, which is often single-phase. However, the most common option is to use the general-purpose three-phase transmission grid, which is the typical configuration in Spain.

As shown in , the electrical system is divided into electrically-isolated singlephased sectors, which are fed from the three-phase network through a transformer.

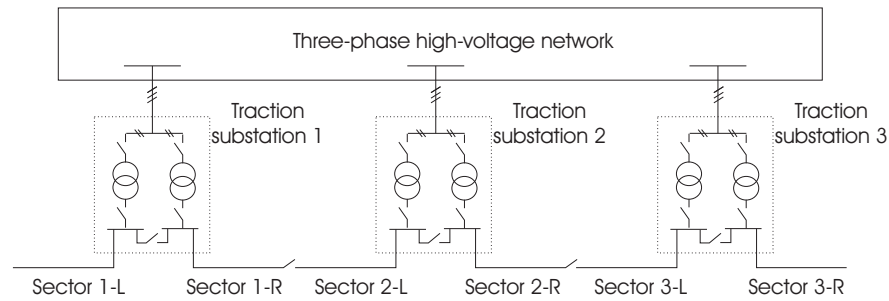

Figure 1: $\quad$ Structure of the power supply system.

Several connections of the transformer are possible as discussed in [1-3], but the most common one is the open-triangle connection (see Figure 2). In this case, transformers are connected between two of the three phases of the high-voltage network.

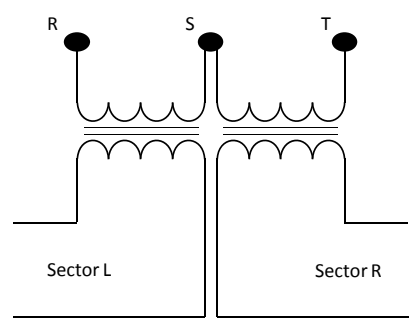

Figure 2: Open-triangle connection of the traction transformer.

As the power consumption of two sectors is not equal, the power consumption seen from the three-phase grid is unbalanced. In order to mitigate the consequences, the open-side phase of the triangle is changed in consecutive substations.

Different procedures exist to calculate the imbalances [4-7]. Analyzing them, it can be established that imbalances depend on: (i) how different are the power consumptions in the sectors fed from the same substation, (ii) the connection type of the transformer and (iii) how small are the direct and inverse sequence short-circuit impedances of the three-phase grid. 
The stronger the network is, the bigger the short circuit power is and the lower the series impedances are. The imbalances due to the connection of highspeed power supply system to the transmission network in strong interconnected three-phase grid are usually small, but have to be analyzed in every case [8].

In weak non-interconnect three-phase grids, such as in islands, imbalances caused by the railways can be a serious problem and have to be analyzed carefully [9]. This paper presents an analysis of this very particular problem, which has not been very extensively treated in the literature. This approach has been applied to a real small isolated power system in which a high-speed railway system is expected to be introduced by the year 2018 .

Section 2 describes the methodology to analyze the impact of a high-speed railway system in a small isolated power system. Section 3 presents an example of the application of the methodology to a real small isolated power system. Finally, Section 4 concludes the paper.

\section{Methodology}

This section describes the methodology proposed to analyze the impact of a high-speed railway system in a small isolated power system. Firstly, the system modelling is detailed. Secondly, the study of the phase to phase imbalances due to the incorporation of a high-speed railway system is described.

\subsection{System modelling}

This subsection presents the model used to study the impact of the high-speed railway system in a small isolated power system and the building of generation and demand scenarios.

\subsubsection{Small isolated power system description: peak and valley scenarios}

In order to calculate the phase to phase imbalances in the small isolated power system the parameters of the buses, lines, transformers and the generators, and the scenarios of generation and demand should be known.

This paper proposes to build two scenarios of generation and demand, one representing a peak hour and the other representing a valley hour. The reason is that in small isolated power systems, the difference between both scenarios can lead to important differences in the results of the phase to phase imbalances.

\subsubsection{Connection of the railway network to the small isolated power system network}

Usually, in non small isolated power systems, the different traction substations are directly connected to different nodes of the power network. Since non small isolated power systems are robust, the imbalance caused in each connection node due to the single-phase load is normally small. However, due to the weakness of small isolated power systems, it seems that it would be better to connect the railway system to the network in a different way. In this paper, two different connections proposed by the infrastructure manager are analyzed: (i) the first connection consists of grouping some of the traction transformers in different 
traction substations and them directly to different nodes of the power network. This first connection of the railway network since it is more common to find it in non small isolated power system will be called non isolated power system connection (NSIPS connection); (ii) the second connection consists of creating a railway network that contains all the traction substations, and connecting this whole network to a single node of the small isolated power system. The imbalances caused in the single connection node to the network will be probably lower since the single-phase loads can be connected inside the railway network alternating their phases of connection and therefore decreasing the imbalances. The second connection of the railway network will be called small isolated power system connection (SIPS connection).

Both connections try to reduce the impact of the railway system in power system network imbalances. This paper proposes to study the effects of both connections in some representative nodes of the small isolated power system and in the railway network. Figure 3 shows the NSIPS and the SIPS connections. In both connections, each transformer of the traction substations feed one sector.
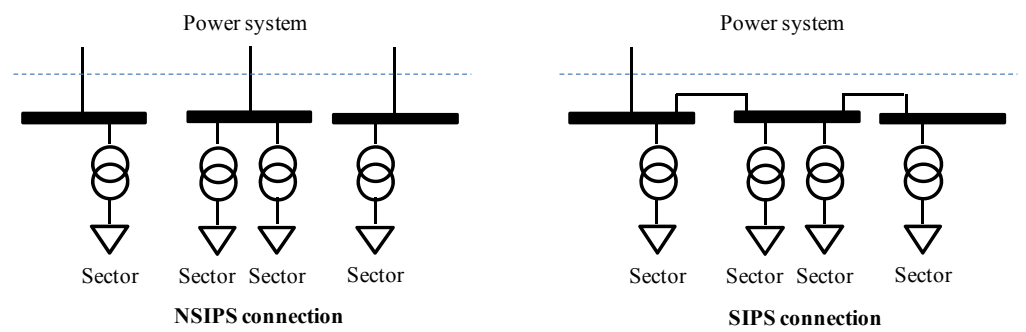

Figure 3: $\quad$ NSIPS and SIPS connections.

\subsection{Study of phase to phase imbalances due to the incorporation of a high-speed railway system in a small isolated power system}

In this subsection the phase to phase imbalances of the voltages of the threephase network due to the incorporation of single-phase loads corresponding to the transformers that feed the catenaries are studied.

Firstly, the procedure to calculate those phase to phase imbalances is detailed. Secondly, the measures of those imbalances that are going to be used in order to analyze the impact in the small isolated power system network and in the railway network are presented.

\subsubsection{Phase to phase imbalances calculation}

To simulate the railway system load (i.e. trains) between two phases, an impedance is connected between the two phases of the three-phase network. The values of $R$ and $X$ of the impedance should be those that involves that the consumption of this impedance is equal to the consumption of the railway system load in each instant. Therefore, the value of the impedance changes with time. 
In order to solve these connections in the three-phase network, the system is decomposed into its symmetrical components (positive, negative, and zero).

Since the connection of the impedance is between two phases, there is no zero component so only the positive and the negative sequences should be solved. For each of those components, the Thévenin equivalent of the system between the two points where the impedance is going to be connected is obtained and the voltages between those points (positive and negative sequence voltages) are calculated. The imbalance in the three-phased voltages is defined as the ratio between the negative voltage and the positive voltage.

\subsubsection{Phase to phase imbalances in the small isolated power system and in the railway network}

From the expected traffic simulations the active and reactive instant power demand of each sector are obtained and therefore, the power in each single-phase transformer. The instant imbalances are then calculated in the representative nodes of the small isolated power network and in the main nodes of the railway network.

After that, the values of the measures required in the phase to phase imbalances standards of the small isolated power system under study are computed. As an example, in Spain, regarding the phase to phase imbalances, there is an internal technical requirement [9] and a European standard [10]. The Spanish technical requirement states [9]: (i) Maximum phase to phase instant imbalance lower that $1 \%$ [Max Ins $(<1 \%)$ ]; (ii) Maximum one minute quadratic moving average of the phase to phase imbalances lower that $0.7 \%$ [Max $1 \mathrm{~min}$ $(<0.7 \%)$ ]. The European standard states [10]: (iii) Maximum ten minutes quadratic moving average of the phase to phase imbalances lower that $2 \%$ [Max 10 min $(<2 \%)$ ]; (iv) Maximum percentage of time in which the ten minutes quadratic moving average of the phase to phase imbalances is over $2 \%$ lower than the $5 \%$, called [ $\mathrm{T} \max 10 \mathrm{~min}(<5 \%)]$.

This procedure is repeated for the different connection modes (NSIPS and SIPS connection) and for the different scenarios proposed (in this paper, peak and valley scenarios).

\section{Case study}

This section presents an example of the application of the methodology proposed in this paper to a real small isolated power system.

\subsection{System description}

This subsection presents the system in which the study of the impact of a highspeed railway system has been made. Since the high-speed railway system is expected to be introduced in year 2018, the expected small isolated power system network of $220 \mathrm{kV}$ and $66 \mathrm{kV}$ for this year has been used.

Table 1 summarizes the number of lines of $220 \mathrm{kV}, 66 \mathrm{kV}$ and $20 \mathrm{kV}$, the number of transformers and the installed power of conventional and renewable generation in the system. 
Table 1: $\quad$ Number of elements and installed power of the network in 2018.

\begin{tabular}{|c|c|c|c|c|c|c|c|c|c|}
\hline \multicolumn{3}{|c|}{ Lines } & \multicolumn{2}{|c|}{ Transformers } & \multicolumn{5}{|c|}{ Generation units } \\
\hline \multirow[b]{2}{*}{$220 \mathrm{kV}$} & \multirow[b]{2}{*}{$66 \mathrm{kV}$} & \multirow[b]{2}{*}{$20 \mathrm{kV}$} & \multirow[b]{2}{*}{$220 / 66 \mathrm{kV}$} & \multirow[b]{2}{*}{$66 / 20 \mathrm{kV}$} & \multicolumn{4}{|c|}{ Conventional Generators } & \multirow{2}{*}{$\begin{array}{c}\text { Renewable generators } \\
\text { Wind, solar and } \\
\text { pumping }\end{array}$} \\
\hline & & & & & Steam & Gas & Diesel & $\begin{array}{c}\text { Combined } \\
\text { cycle }\end{array}$ & \\
\hline 15 & 67 & 6 & 19 & 37 & 4 (287MVA) & 6 (234MVA) & 2 (55MVA) & 3 (792MVA) & (643MW) \\
\hline
\end{tabular}

Two possible scenarios of generation and demand for the year 2018 have been estimated, one represents a peak hour and the other represents a valley hour. The active and reactive power amount to $757 \mathrm{MW}$ and $215 \mathrm{MVAR}$ in the peak scenario and to $357 \mathrm{MW}$ and 101MVAR in the valley scenario.

\subsection{Railway system loads}

The high-speed railway network under study consists of three sectors fed by three different transformers connected to two traction substations (traction substation A and traction substation B).

From the expected traffic simulations the active and reactive instant ( 5 seconds) power demand of each sector are obtained for the peak and the valley scenarios. As an example, Figure 4 shows the active power demand during the peak scenario for each sector. In a dash line the quadratic average of the power has also been drawn.
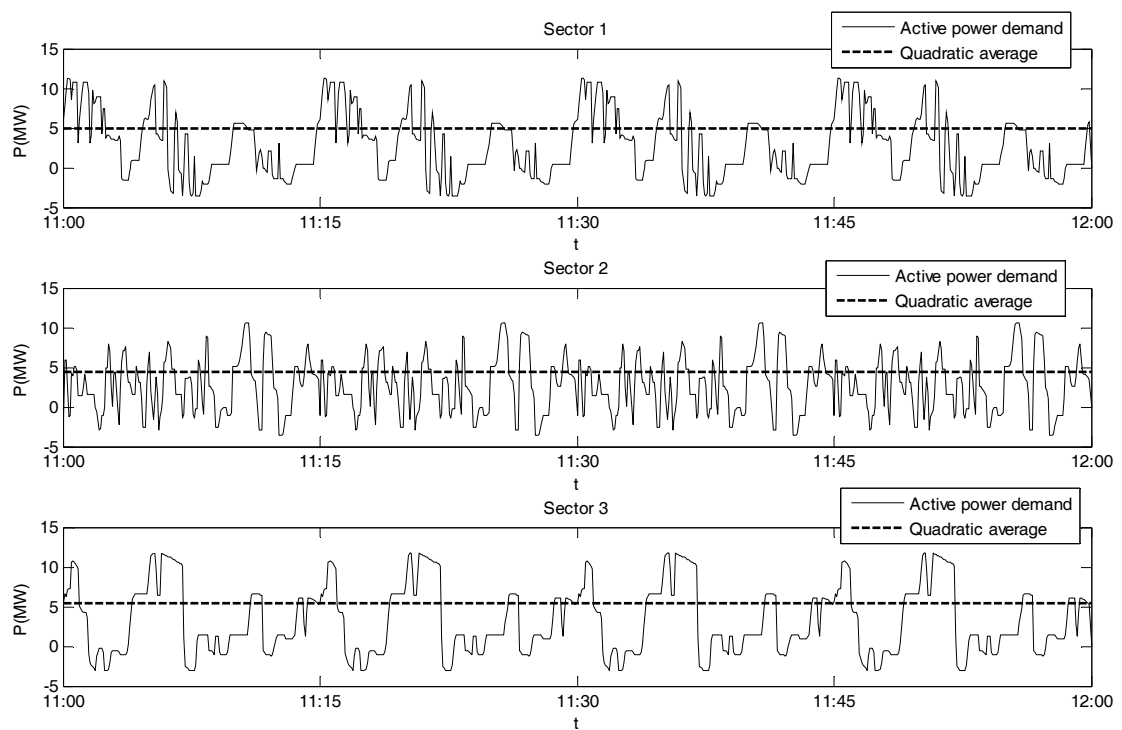

Figure 4: $\quad$ Active power demand in the peak scenario.

Note that the expected traffic schedule has a 15 minute period and that, in most instances, power demand is different in all three sectors. 
The railway network of this case-study includes transport interchanges, tunnels, passenger stations, and two traction substations. The demand of those elements varies during the day. For the peak scenario, the active demand of the sectors (corresponding to the sum of the quadratic average of the power of all the sectors) amounts to $14.8 \mathrm{MW}$ while the active and reactive demand of the other elements of the railway system (tunnels, stops, etc) amount to $8.2 \mathrm{MW}$ and 4.6MVAR. For the valley scenario, the active demand of the sectors amount to $14.6 \mathrm{MW}$ while the active and reactive demand of the other elements of the railway system amount to $7.3 \mathrm{MW}$ and 4.6MVAR.

\subsection{Connection of the railway network to the small isolated power system network}

As previously mentioned, two different connections of the railway system to the network are going to be studied: the NSIPS and the SIPS connection.

In the upper part of Figure 5 the NSIPS connection is represented. It can be observed that each of the traction substations is connected to two different nodes of the small isolated power system network (Node 1: Connection node Substation A and Node 2: Connection node Substation B). In the lower part of the SIPS connection is represented. The whole railway network with both
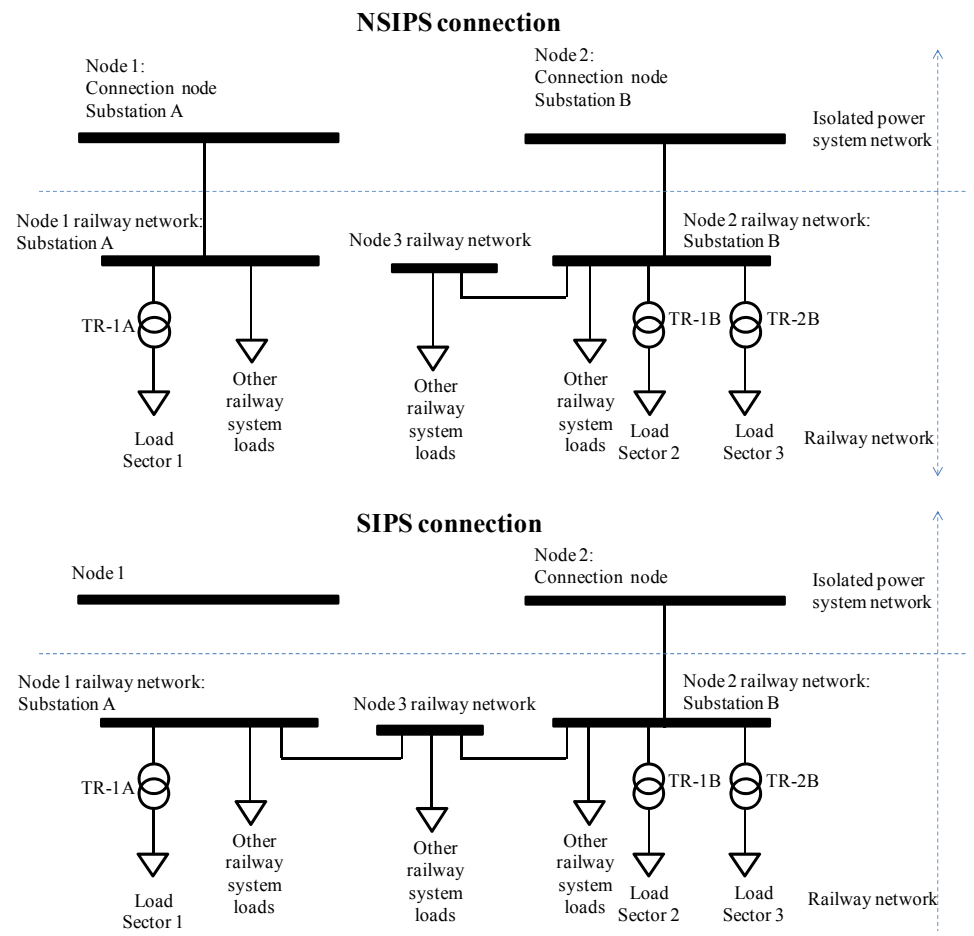

Figure 5: $\quad$ NSIPS and SIPS connections. 
traction substations is connected to a single node of the small isolated power system network (Node 2: Connection node).

As told in subsection 2.2.2, representative nodes of the isolated power system and of the railway network should be used to study the imbalances. The representative nodes of the isolated power system network include the nodes where the railway system is connected to the network (Connection node, $\mathrm{CN}$ ).

The representative nodes of the railway network should include those nodes where the train loads are connected which are Node 1: Substation A and Node 2: Substation B and the nodes in which the railway network is connected to the small isolated power system network (in this study-case is the same Node 1: Substation A and Node 2: Substation B if the NSIPS connection is used and only the Node 2: Substation B if the SIPS connection is used). In addition, another node between both substations called Node 3 has been added to model other loads.

\subsection{Instantaneous phase to phase imbalances in the small isolated power system network}

Figure 6 represents the value of the imbalances every five seconds of the hour in every representative node during the peak and the valley scenario for the NSIPS connection. Node 1 and Node 2 are the nodes where the railway system is connected to the network. Node 3 and Node 4 are other representative nodes of the system.
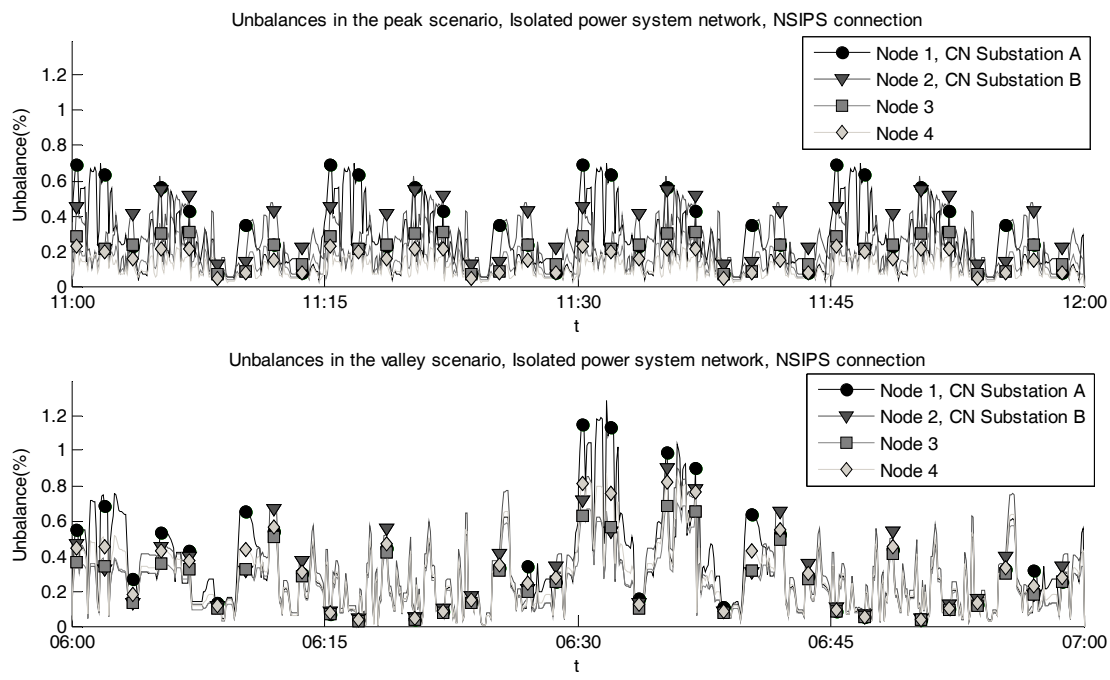

Figure 6: NSIPS connection imbalances in the small isolated power system.

From Figure 6, it can be observed that the imbalances are higher at the nodes where the railway network is connected to the small isolated power system network (Node 1 and Node 2). It can also be observed that in Node 3 and in 
Node 4, the phase to phase imbalances are quite similar. Regarding the differences between the peak and the valley scenarios, it should be mentioned that the imbalances during the peak scenario have lower variability.

Figure 7 represents the value of the imbalances every five seconds during the peak and the valley scenario for the SIPS connection. Node 2 is the node where the railway system is connected to the network. Node 1, Node 3 and Node 4 are other representative nodes of the system.

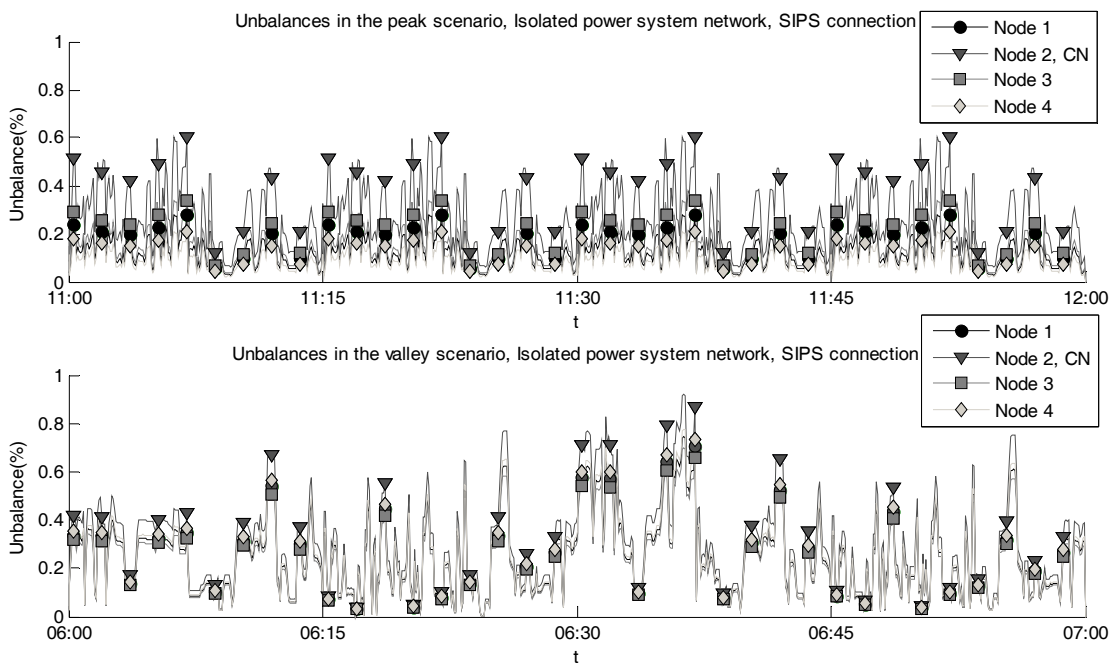

Figure 7: SIPS connection imbalances in the small isolated power system.

The same conclusions as the one obtained with the NSIPS connection are obtained.

From both figures it can be observed that the imbalances produced in Node 1 with the NSIPS connection are much higher than with the SIPS connection. However, the imbalances produced in Node 2 are similar.

\subsection{Instantaneous phase to phase imbalances in the railway network}

Since results of the NSIPS connection in the railway network are going to be very similar to the one obtained in the analogous nodes of the power system network, in Figure 8 only the value of the instant imbalances of the peak and the valley hours of every representative node of the railway network in the SIPS have been represented.

From it can be observed that the node of the railway network in which the imbalance is higher is the Node 1 in which there is a traction transformer but is far away from the node in which the railway network is connected to the small isolated power system network (Node 2: Substation B). This fact is explained because Node 2 has a higher short-circuit power in the peak and valley scenario since it is connected to the small isolated power system. Phase to phase 

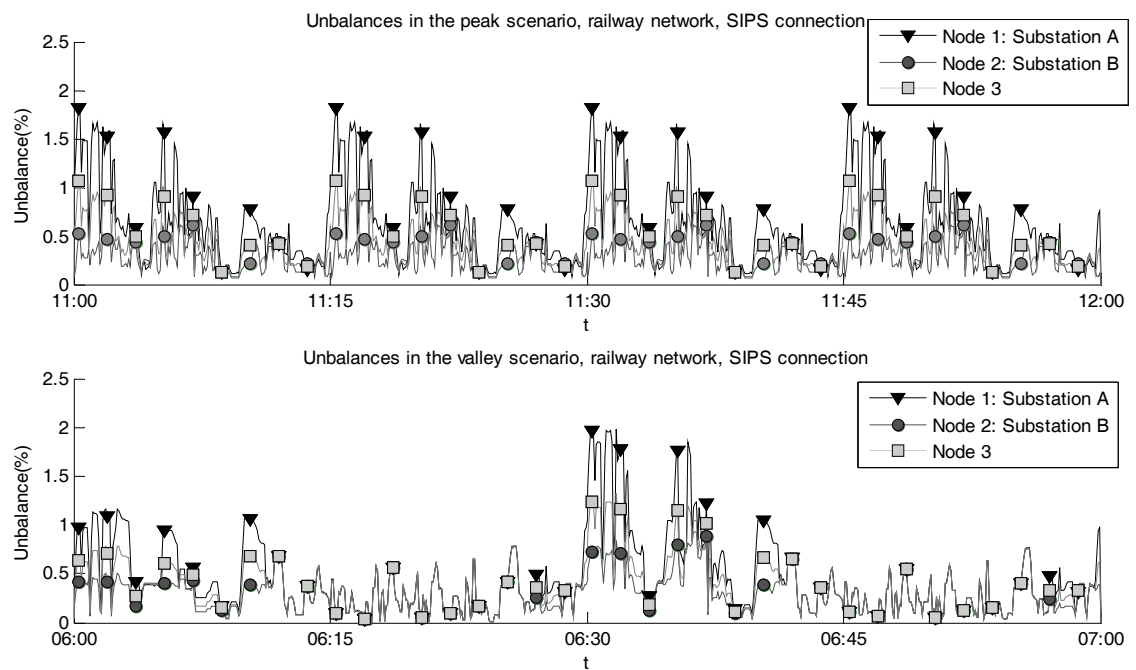

Figure 8: $\quad$ SIPS connection imbalances in the railway network.

Table 2: $\quad$ Short-circuit powers in the SIPS connection.

\begin{tabular}{|c|c|c|}
\cline { 2 - 3 } \multicolumn{1}{c|}{} & \multicolumn{2}{c|}{ Scc (MVA) } \\
\cline { 2 - 3 } \multicolumn{1}{c|}{} & Peak & Valley \\
\hline Node 1:Subs tation A & 579 & 474.1 \\
\hline Node 2:Subs tation B & 1880 & 1161.4 \\
\hline
\end{tabular}

imbalances have a high relation with the short-circuit power as they are roughly inversely proportional to the short-circuit power of the connection node. Shortcircuit power for Node 1 and Node 2 in peak and valley scenarios are presented in Table 2.

\subsection{Standards measures for phase to phase imbalances}

Table 3 presents the maximum value achieved for each of the standard measures for the main representative nodes of the small isolated power system network and of the railway network. The results for the NSIPS and the SIPS connection and the peak and valley scenarios are included.

In it can be observed that regarding the small isolated power system network, the values of the three standards measures obtained in Node 1 of the isolated power system network with the NSIPS connection are much higher than with the SIPS connection. However the values of measures in Node 2 are similar. Regarding the railway network, the situation is the opposite, the three measures of the standards of the Node 1: Substation A are higher with the SIPS connection while in the Node 2: Substation B are quite similar. 
Table 3: $\quad$ Maximum value of the standard measures of the imbalances.

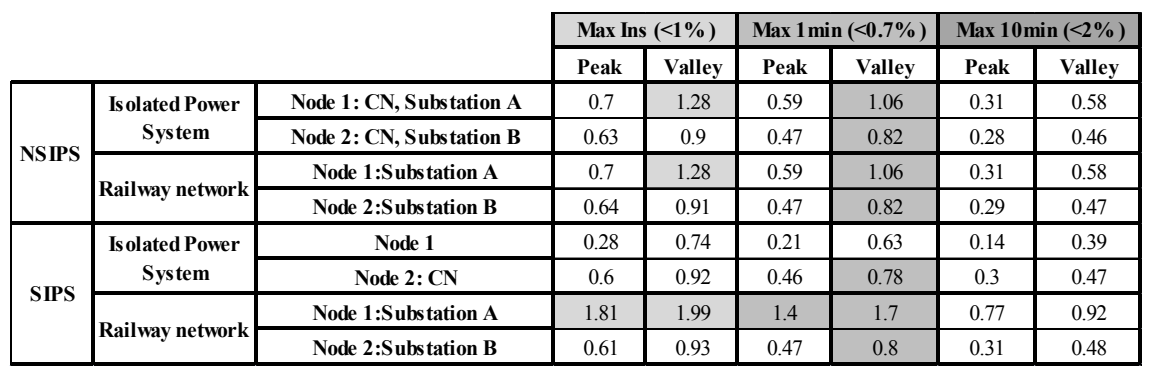

It can also be observed that the [Max Ins $(<1 \%)]$ measure is not complied in the isolated power system network in the Node 1: CN substation A in the valley hour scenario if the NSIPS connection is used. This measure is not complied in the railway network in the Node 1 railway network: Substation A if the SIPS connection is used in the peak and in the valley hour scenarios. However, in Table 3 it can be observed that the $[\operatorname{Max} 1 \min (<0.7 \%)]$ measure is not complied in all the connection cases (NSIPS and SIPS) in all the nodes if the valley scenario is studied (excepting Node 1 of SIPS connection).

Regarding the [Max 10min $(<2 \%)]$ measure, it is complied in all the connection configurations in all the nodes if the valley and the peak scenarios are studied. Following this result, the $[\mathrm{T} \max 10 \mathrm{~min}(<5 \%)]$ measure is always zero.

Since the imbalances standards mainly affect the small isolated power system networks, SIPS configuration is more appropriated.

\section{Conclusions}

This paper has presented the analysis of imbalances in the three-phase network of a small isolated power system due to the connection of the power supply system of AC-fed high-speed railways fed. Due to the weakness of the grid, imbalances can be a serious problem that has to be analyzed.

The analysis highlights the vulnerability of small isolated power systems. It also evidences the importance of using peak and valley scenarios since clearly different results of the imbalances are obtained. The analysis allows choosing the connection of the power supply system to the three-phase network that causes fewer imbalances.

Results show that the imbalances are higher at the nodes where the power supply system is connected to the small isolated system. If peak and valley scenarios are used, results show that the imbalances during the peak scenario have lower variability and that the maximum values of the standards measures are always obtained in the valley scenario.

In general, values of the standards measures in the nodes of the small isolated power system network are higher with the NSIPS connection than with the SIPS connection. However, values of those measures in the railway network are in general higher with the SIPS connection than with the NSIPS connection. Since 
the imbalances standards mainly apply to the small isolated power system network, SIPS configuration may be preferred.

\section{References}

[1] H. Erfanian Mazin and Wilsun $\mathrm{Xu}$, "An investigation on the effectiveness of Scott transformer on harmonic reduction," in Power and Energy Society General Meeting - Conversion and Delivery of Electrical Energy in the 21st Century, 2008 IEEE, 2008, pp. 1-4.

[2] N. Golovanov, G. C. Lazaroiu, M. Roscia and D. Zaninelli, "Voltage unbalance vulnerability areas in power systems supplying high speed railway," in Power Engineering Society General Meeting, 2005. IEEE, 2005, pp. 2509-2514 Vol. 3.

[3] T. Chen, "Comparison of Scott and Leblanc transformers for supplying unbalanced electric railway demands," Electric Power Systems Research, vol. 28, pp. 235-40, Jan, 1994.

[4] Liu Yu-Quan, Wu Guo-Pei, Hua Huang-Sheng and Wang Li, "Research for the effects of high-speed electrified railway traction load on power quality," in Electric Utility Deregulation and Restructuring and Power Technologies (DRPT), 2011 4th International Conference on, 2011, pp. 569-573.

[5] M. Aeberhard, C. Courtois and P. Ladoux, "Railway traction power supply from the state of the art to future trends," in Power Electronics Electrical Drives Automation and Motion (SPEEDAM), 2010 International Symposium on, 2010, pp. 1350-1355.

[6] P. Heine, M. Hyvärinen, J. Niskanen, A. Oikarinen, H. Renner and M. Lehtonen, "The impact of $50 \mathrm{hz}$ railroad systems on utility system unbalance," in Electric Power Quality and Supply Reliability Conference (PQ), 2010, 2010, pp. 61-66.

[7] Hung-Yuan Kuo and Tsai-Hsiang Chen, "Rigorous evaluation of the voltage unbalance due to high-speed railway demands," Vehicular Technology, IEEE Transactions on, vol. 47, pp. 1385-1389, 1998.

[8] R. Barnes and K. T. Wong, "Unbalance and harmonic studies for the Channel Tunnel railway system," Electric Power Applications, IEE Proceedings B, vol. 138, pp. 41-50, 1991.

[9] G. Astengo, C. Rossi and G. Sciutto, "Single-phase $25 \mathrm{kV}$ electrification of Sardinia railway: Some particular problems related to an electrified railway located in an island," in Main Line Railway Electrification, 1989., International Conference on, 1989, pp. 178-181.

[10] Red Eléctrica de España TI.E/02/040-2010 "Facilities connected to the mainland transmission network: minimum requirements for design and equipment".

[11] CENELEC EN 50160:11-1999 "Voltage characteristics of electricity supplied by public distribution systems". 